\title{
Diagnosis and Treatment of Supraventricular Tachyarrhythmia in Pediatric Population: a Review Article
}

\author{
Hany MA Abo Hadeed MD* \\ Department of Pediatrics, Taibah University, Saudi Arabia
}

Submission: October 04, 2016; Published: January 30, 2017

*Corresponding author: Hany MA Abo Hadeed, Assistant professor of pediatrics and pediatric cardiology, Department of Pediatrics, Faculty of medicine, Taibah University, Al-Madinah Al-Munawwarah, Saudi Arabia, Tel: +966568953372; Fax: +966568985102;

Email: hmabohadid@taibahu.edu.sa

\begin{abstract}
Supraventricular tachyarrhythmia (SVT) is the most common tachyarrhythmia in pediatric patients and accounts for more than $90 \%$ of pediatric arrhythmias. As many as 16 different mechanisms of pediatric SVT exist; we focused in this review article on the three most common groups of SVT mechanisms in pediatrics: atrioventricular re-entry tachyarrhythmia (AVRT), atrioventricular nodal re-entry tachyarrhythmia (AVNRT), and a trial tachyarrhythmia (AT). Many methods have been implicated in the diagnosis of SVT like full history taking regarding onset and frequency of palpitation, syncope, chest pain, dispend, heart failure, drug intake and physical examination. Children with suspected SVT must be subjected to resting 12-lead surface ECG and ECG during the attack. Echocardiography must be done for detection of any structural heart diseases. Moreover, recent techniques like Electro physiologic study (EPS) is the most definitive diagnostic procedure. Many therapeutic strategies like drugs and radiofrequency ablation (RFA) have been succeeded to provide a significant reduction of the risk of SVT, but RFA offers the prospect of cure of the arrhythmia and avoidance of drug-associated side effects. It is often becoming the first line of therapy for many children with SVT, as it has a relatively low morbidity and mortality, and it results in a low rate of recurrence of SVT.
\end{abstract}

Keywords: SVT; Children; Diagnosis; Treatment

Core Tip: This manuscript discusses the most common types, recent strategies in diagnosis and management of Supraventricular tachycardia in pediatric age group.

\section{Introduction}

Supraventricular tachyarrhythmia (SVT) is the most symptomatic pediatric arrhythmia resulting from an abnormal mechanism that requires structures in the heart above the bifurcation of the bundle of His for its continuation. Clinical symptoms of SVT are variable; ranging from asymptomatic or minor palpitations to more severe manifestations [1]. The incidence of SVT has been documented to affect between 1 in 250 to 1 in 25,000 children [2].

Variable mechanisms of SVT exist; we concentrated on the three commonest forms occurring in children which are: atrioventricular re-entry tachyarrhythmia (AVRT), atrioventricular nodal re-entry tachyarrhythmia (AVNRT), and a trial tachyarrhythmia (AT) [3].

\section{Most Common Types of Svt in Pediatrics}

Atrioventricular re-entrant tachyarrhythmia (AVRT) or accessory pathway re-entry tachyarrhythmia.
An accessory pathway (AP) is a microscopic bridge of muscle connecting atrium and ventricle that bypasses the normal electrical insulation of the AV ring. The impulse spreads down either via the atrioventricular (AV) node or the AP and then up the other. A re-entrant unconventional circuit is formed, [4] (Figure 1).

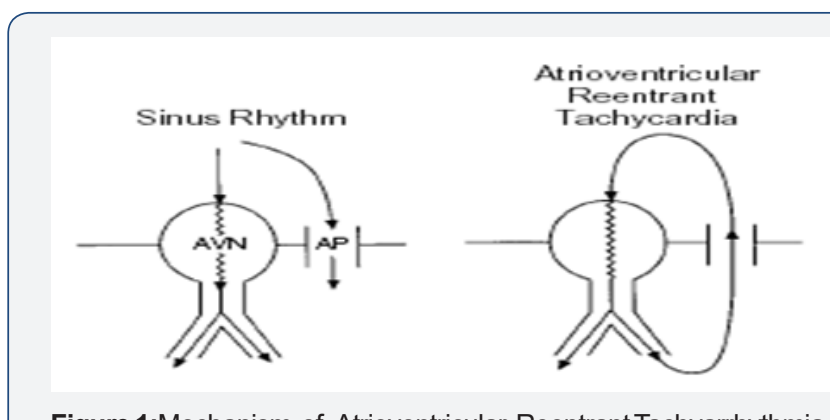

Figure 1:Mechanism of Atrioventricular Reentrant Tachyarrhythmia. 
AVRT is the commonest type of SVT in the pediatric population forming two-thirds of the affected children, then comes the atrioventricular nodal reentry tachyarrhythmia (AVNRT) and the trial tachyarrhythmia (AT) [5]. AVRT is more common in males, but its incidence decreases with age. On the other hand, the incidence of AVNRT and a trial tachyarrhythmia is increased with age [6].

\section{Atrioventricular nodal reentry tachyarrhythmia (AVNRT)}

The AV node is composed of "slow" pathway and "fast" pathway. AVNRT happens when an ante grade impulse is blocked at one pathway (usually the fast pathway), resulting in conduction over the other (usually the slow pathway). After the impulse is travelled down via the slow pathway, the fast pathway is now unblocked and the impulse can spread retrograde via the fast pathway. So, a re-entrant circuit is formed, [7] (Figure 2).

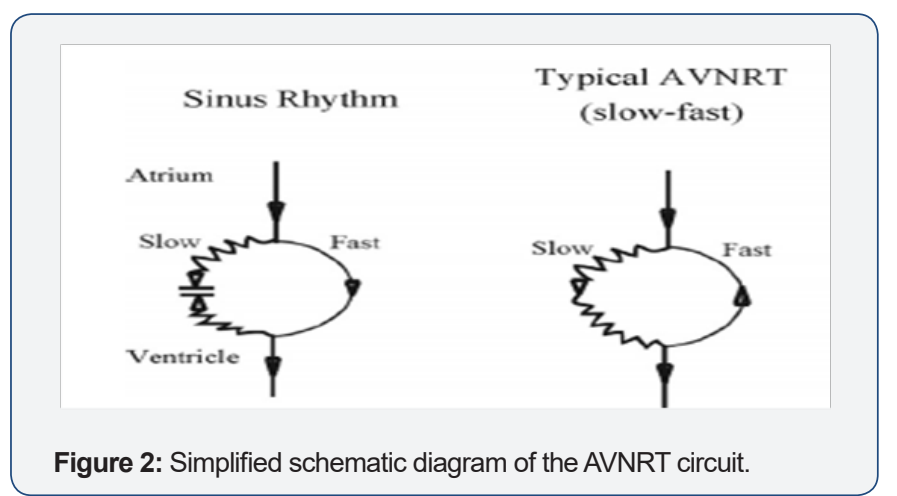

AVNRT accounts for $15 \%$ of cases of pediatric SVT, mostly present over the age of 5 years and almost completely absent in infants. Females were more than males in cases diagnosed as AVNRT or AT [6]. Atria tachyarrhythmia (AT). AT is mostly due to abnormal automaticity, may be formed by remnant embryonic cells with automatic qualities, causing abnormal impulses that come away from normal sinus node but still within the atria. Although it is a rare condition, but still the third common mechanism of tachyarrhythmia occurring in children, if remains undetected can result in a dilated cardiomyopathy [7].

\section{History and clinical presentations of Svt in pediatrics}

The clinical presentation varies with the age of the patient; generally, it differs in infancy from that in an older child. In infancy, most attacks occur before 6 months of age, and may often less than 4 months. They may be undetected or associated with pallor. The parents may take their child to the doctor with a complaint of poor feeding, or vomiting. The rapid breathing or ashen color may not be observed by them. By the time, the pediatrician sees them: there is usually significant cardio-respiratory distress. The infant will often have signs of heart failure. Occasionally frank circulatory shock will be present [8]. Occasionally, a young patient may have palpitations, chest or abdominal pain, occasionally syncope, particularly with exertion may occur, but the presence of congestive heart failure beyond infancy is extremely uncommon [9](Table 1).
Table 1: Treatment strategy in SVT [15].

\begin{tabular}{|c|}
\hline 1. Identify Mechanism of SVT \\
a)AVRT \\
b)AVNRT \\
c)AT \\
\hline 2. Acute Termination of Established SVT Attack \\
a)Vagal maneuvers' \\
b)Adenosine IV \\
c)Electrical cardioversion \\
i.Direct current (DC) shock \\
ii.Via Transesophageal \\
3. Chronic Management \\
a)General considerations \\
i.Type of SVT mechanism, age of presentation, Severity of \\
symptoms. \\
b)Treatment options \\
ii.Periodic pharmacologic therapy \\
ii.Daily pharmacologic treatment \\
Observation and documentation of symptoms \\
a treatment \\
\hline
\end{tabular}

\section{Physical Examination of Svy in Pediatrics}

Following the history, the physical examination should be performed: unexplained hypothermia, with poor perfusion, a picture of sepsis but with negative cultures. In the older infants, irritability associated with vomiting and sometimes diarrhea (uncommon). In approximately $20 \%$ of infants, SVT is detected during the routine examination without symptoms [10].

Auscultation should be done to reveal the presence of structural heart disease, heart rate and rhythm should be noted. Further evaluation will depend on the severity and frequency of symptoms, the age of the child, and the presence of structural heart disease. Infants with SVT may have a heart rate range from 220 to 300 BPM. Older children generally have slower rates which may count from 180 to $240 \mathrm{Bpm}$. Untreated SVT can result in congestive heart failure (CHF) within 24 to 48 hours. Termination of the attack by vagal maneuvers may suggest a reentrant tachyarrhythmia involving AV node [5].

\section{Diagnostic investigations of Svt in pediatrics}

Recording a 12- Lead Electrocardiography (ECG) at rest should be done and examined for any abnormal rhythm, delta waves, abnormal QT interval, sinus tachyarrhythmia, or any sign of underlying structural heart disease. [11]. ECG can clearly diagnose about $80 \%$ of AVNRT and AVRT, but incorrectly categorize approximately $20 \%$ of cases of SVT; therefore, the ECG cannot serve as the sole means for determining the mechanism of tachyarrhythmia [12].

Another diagnostic tool is worn by the patient named Holter monitor. It can record continuous ECG tracing for 24 to 48 hours, 
having the same idea as classic ECG wires and electrodes but it is a portable device that provides information about symptoms that can occur during the day [7].

In selected patients with occasional complaints (i.e. less than two episodes of SVT per month) and associated with disabling symptoms (i.e. hemodynamic instability) can be diagnosed by implantable loop recorders. If the clinical history is not enough or other measures have failed to identify the SVT mechanism, then recordings and stimulation can be done via Tran esophageal a trial loops for these patients for diagnosis or to provoke paroxysmal tachyarrhythmia [13].

The possibility of structural heart disease should be excluded by Echocardiography examination in children with identified attacks of SVT, which is not possible to be detected via physical examination or resting 12-lead ECG [14].

Other investigations can be done to exclude other causes included in the differential diagnosis of SVT. Chest x-ray with lateral and anteroposterior views is done to diagnose cardiomyopathy and CHF. Laboratory tests which include; serum electrolytes to diagnose any imbalance leading to abnormal cardiac rhythm, complete blood cell count (CBC) with differential to exclude infections or anemia, also, screening of toxic substances, thyroid function tests, and arterial blood gas can be helpful [5]

The most accurate diagnosis can be done with the electrophysiological study (EPS). It is used for clear classification of different mechanisms of SVT. Also, EPS combined with catheter ablation can be used as a definitive long-term therapy [7].

\section{Management of Svt in Pediatric Age Group}

The dealing with SVT can be done in two ways: acute termination of the attack of tachyarrhythmia and prevention of recurrences (chronic therapy). The acute treatment begins with documentation of the arrhythmia with a 12 lead ECG, assessment of hemodynamic status and recognition of the arrhythmia mechanism. Chronic therapy is based on the SVT mechanism, the patient's age, and the frequency and patient's complaint during the attack as well as access to medical care and sophistication of the patient caretaker (Table) [15].

\section{Acute management of Supraventricular tachyarrhythmia}

For the aerodynamically tolerated episode, vagal physical maneuvers are to be used first. The patient may be taught to do Valsalva maneuver (the most potent physical maneuver), induce vomiting, dive the face in the iced water for 10-20 seconds, or carotid sinus massage may be done, an ocular pressure is not recommended for children. These physical maneuvers are potent in terminating re-entry SVT; mostly AVRT and to less extent AVNRT [11].

An ultra-short-acting drug (Adenosine) is highly effective in terminating AV node-dependent SVT, but it is of limited value in the diagnosis of AT. Vagal maneuvers and adenosine produce transient AV node block and result in sudden termination of SVT in any tachyarrhythmia involving AV node but not in AT [16].

The use of Beta blockers or long-acting calcium channel blockers is of value especially for patients with frequent a trial or ventricular premature beats which may act as a cause of recurrence of SVT but they must be used with great caution as they may potentiate hypotension [11].

In an aerodynamically unstable child with severe hypotension, electrical cardio version by synchronized DC shocks is the treatment of choice. It is a safe and effective procedure in the majority of patients [2].

\section{Long term (chronic) management of Supraventricular tachyarrhythmia}

The decision to initiate chronic therapy in infants and children is based on symptoms, efficacy rates, safety issues, expected response to therapy and frequency of episodes. The range of therapeutic options aimed at treating disorders of heart rhythm has expanded tremendously over the past decade; the complexity of actions produced by ant arrhythmic agents occasionally leaves the clinician frustrated by attempted at predicting the clinical results [2].

A child with SVT should be viewed globally while putting a long-term therapeutic plan, as this may affect him through his lifelong. A pharmacologic therapy is used to provide symptom relief. The usual selection of drugs (in order) is propranolol, verapamil and amiodarone. Dioxin is especially considered in cases with AVNRT, while amiodarone in combination with propranolol or/ and verapamil is effective in cases with AT [11].

Radiofrequency ablation (RFA) using the traditional radiofrequency energy to heat and destruct the site of origin of the SVT mechanism has been more widely applied in children since the 1990s [3]. It is now considered as the first line of management for many children with SVT and offering the prospect of cure of the arrhythmia and avoidance of drug-associated side effects. Success rates for ablation are as high $95 \%$ and characterized by low morbidity, low mortality, and a low rate of SVT recurrence [17]. RFA may have some complications resulting from the vascular access (i.e. hematomas, perforation of the aorta, deep venous thrombosis), or during catheter manipulation (i.e. alular damage, perforation of the coronary sinus or myocardial wall), or inaccurate ablation (resulting in i.e. AV node block, myocardial perforation, coronary artery spasm, transient ischemic attacks) [18]. Successful elimination of SVT substrate results in improving the pediatric quality of life regarding physical, emotional, social, school and psychosocial functions [19].

In conclusion, SVT is a common pediatric age group problem. Many methods have been implicated in the diagnosis of SVT like detailed history taking and physical examination. ECG and echocardiography must be done to children suspected to have SVT. Electro physiologic study (EPS) is a recent technique for definitive diagnosis. RFA is a definitive therapy for many children with SVT. However, further studies are mandatory in order to provide 
more novel therapeutic agents for complete protection of SVT and decreasing the complications of already used management procedures.

\section{Acknowledgement}

The author is grateful for Deanship of Scientific Research (DSR), Taibah University and Medina, Saudi Arabia for support.

\section{Author Contributions}

Abo Hadeed H.M.A. did the literature review and analysis, wrote the paper; drafting and critical revision and editing, and final approval of the final version.

\section{Open-Access}

This article is an open-access article which was selected by an in-house editor and fully peer-reviewed by external reviewers. It is distributed in accordance with the Creative Commons Attribution Non Commercial (CC BY-NC 4.0) license, which permits others to distribute, remix, adapt, build upon this work non-commercially, and license their derivative works on different terms, provided the original work is properly cited and The use is non-commercial. See: http://creativecommons.org/ licenses/by-nc/4.0/

\section{References}

1. Chiu SN, Lu CW, Chang CW, Chang CC, Lin MT, et al. (2009) Radiofrequency catheter ablation of Supraventricular tachyarrhythmia in infants and toddlers Circ J 73(9): 1717- 1721.

2. Chun TU, Van Hare GF (2004) Advances in the approach to treatment of Supraventricular tachyarrhythmia in the pediatric population. Carr Cardio Rep 6(5): 322-326.

3. Hafez, MM. Abu-Elk heir, M. Shakier, HF. Al-Marsafawy, HM. Abo-Haded et al. (2012) Intermediate Term Follow Up Results. Clin Med Insigne Cordial 6: 7-16.

4. Lad sans EJ, (2009) Diagnosis, evaluation and treatment of cardiac arrhythmias. Pediatr Child Health. 1: 30-36.

5. Doniger SJ, Sheriff GQ (2006) Pediatric dysrhythmias. Pediatr Clin North Am Pediatr 53 (1): 85-105.

6. Anand RG, Rosenthal GL, Van Hare GF, Snyder CS (2009) Is the mechanism of Supraventricular tachyarrhythmia in pediatrics influenced by age, gender or ethnicity? Conge nit. Heart Dis 4(6): 464468.

7. Schlechte EA, Boramanand N, Funk M, (2008) Supraventricular Tachyarrhythmia in the Pediatric Primary Care Setting: Age related
Presentation, Diagnosis, and Management. J Ped Health Care 22(5): 289-299.

8. Balaguer Gargallo M, Jordán García I, Caritg Bosch J, Cambra Lasaosa FJ, prada Heremogenes, et al. (2007) Supraventricular tachyarrhythmia in infants and children Ann Pediatr 67(2): 133-138.

9. Weinstein S, Cua C, Chan D, Davis J (2003) Outcome of symptomatic patients undergoing extra cardiac Font an conversion and cry ablation. J. Thorac. Cardiovasc Surg 126(2): 529-536.

10. Gulati S, Kalra V (2003) Stroke in children. Indian J Pediatr 70(8): 639648.

11. Blomström-Lundqvist C, Scheinman MM, Aliot EM, Alpert JS, Calkins $\mathrm{H}$ Camm AJ, et al. (2003) ACC/AHA/ESC guidelines for the management of patients with Supraventricular arrhythmias--executive summary. a report of the American college of cardiology/American heart association task force on practice guidelines and the European society of cardiology committee for practice guidelines (writing committee to develop guidelines for the management of patients with Supraventricular arrhythmias) developed in collaboration with NASPE-Heart Rhythm Society. J Am Coll Cardiol 42(8): 1493-1531.

12. Snyder CS, Fenrich AL, Friedman RA, Rosenthal G, Kertesz NJ, (2003) Usefulness of echocardiography in infants with Supraventricular tachyarrhythmia. Am J cardiol 91(10): 1277-9.

13. Melton PE, Zlojutro M, Kimminau K, Crawford MH, (2006) Biological aging and Cox hazard analysis of mortality trends in a Mennonite community from south-central Kansas. Am J Hum Biol X 18(3): 387401.

14. Emmel M, Sreeram N, Schickendantz S, Brockmeier K (2006) Experience with an ambulatory 12-lead Holter recording system for evaluation of pediatric dysrhythmias J Electrocardiol 39(2): 188-193.

15. Lee KW, Badhwar BS, Scheinman MM, (2008) Supraventricular tachyarrhythmia- Part II: History, Presentation, Mechanism, and treatment. Curr Probl Cardiol 33(10): 557-622

16. Lee PC, Hwang B, Chen SA, Tai CG, Chen YJ, et al. (2007) The results of radiofrequency catheter ablation of Supraventricular tachyarrhythmia in children. Pacing Clin Electrophysiology 30(5): 655-661.

17. Vaksmann G, D'Hoinne C, Lucet V, Guillaumont S, Lupoglazoff J, et al. (2006) Permanent junctional reciprocating tachyarrhythmia in children: A multicenter study on clinical profile and outcome Heart 92(1): 101-104.

18. Van Hare GF, Javitz H, Carmelli D, Saul JP, Tanel RE, al. (2004) Prospective assessment after pediatric cardiac ablation: Demographics, medical profiles, and initial outcomes. J Cardiovasc Electrophysiology 15(7): 759-770.

19. Striper M, Leong T, Bajaj T, Huckaby J, Frias P,et al. (2010) Does ablation of Supraventricular tachyarrhythmia in children with a structurally normal heart improve quality of life? Congenit Heart Dis 5(6): 587-593.

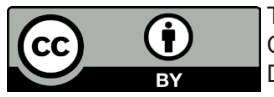

his work is licensed under Creative Commons Attribution 4.0 Licens DOI: 10.19080/AJPN.2017.02.555595

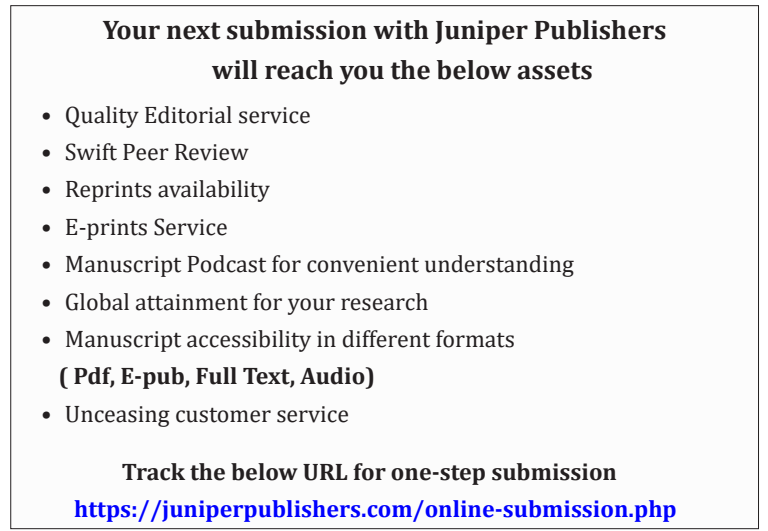

